\title{
Association of clinical sub-phenotypes and clinical deterioration in COVID-19: further cluster analyses
}

\author{
Michiel Schinkel ${ }^{1 *} \mathbb{D}$, Brent Appelman ${ }^{1}$, Joe Butler ${ }^{1}$, Alex Schuurman ${ }^{1}$ and W. Joost Wiersinga ${ }^{1,2}$ on behalf of the \\ COVID Predict Study Group
}

(c) 2021 Springer-Verlag GmbH Germany, part of Springer Nature

\section{Dear Editor,}

Coronavirus disease 2019 (COVID-19) presents in various ways [1]. Recently in the journal, Legrand and colleagues identified three distinct clinical sub-phenotypes of COVID-19, which may help recognize patients at high risk of deterioration [2]. Earlier work in sepsis has shown that clinical phenotypes may help understand the heterogeneity in disease presentation and inform trial design [3, 4]. The retrospective cohort study of Legrand et al. consisted of 893 patients of which 608 were used for cluster analysis, after excluding patients with missing data. Their thorough selection yielded 22 candidate variables for cluster analysis, including disease history, demographics, symptoms and concomitant medication.

We aimed to validate the findings by Legrand et al. in our Dutch CovidPredict cohort. This cohort consisted of COVID-19-positive patients admitted to ten teaching hospital across the Netherlands. COVID-19 was defined as a positive SARS-CoV-2 PCR or CORADS score of at least four [5]. Patients were included between 27 February and 4 December 2020. Approval was granted by the Institutional Review Board of the Amsterdam University Medical Centers (20.131).

\footnotetext{
*Correspondence: m.schinkel@amsterdamumc.nl

${ }^{1}$ Center of Experimental and Molecular Medicine (C.E.M.M.), Location Academic Medical Center, Amsterdam UMC, Amsterdam, The Netherlands

Full author information is available at the end of the article
}

We included 2019 patients and used similar candidate variables and number of clusters as Legrand et al. [2] (see variables in the supplementary file). In total, 657 patients were treated in the intensive care unit (ICU) or died during the following 21 days of COVID-19. Three sub-phenotypes were identified, which are presented in Fig. 1 (see supplementary Table 1 and 2 for baseline characteristics, and Figure 1-3 for cluster characteristics).

Sub-phenotype $1 \quad(n=592)$ mainly included young (median age $63[\mathrm{IQR}=53-74])$ females $(74.5 \%)$, characterized by a high prevalence of gastro-intestinal complaints (84.3\%) and sputum production (63\%). Comorbidities and medication usage were scarce. The composite outcome of ICU admittance/death rates was relatively low compared to the other groups (24.7\%).

Sub-phenotype $2 \quad(n=876)$ included more males (80.4\%) with a median age of $63[\mathrm{IQR}=53-73.1]$ years, few comorbidities and the lowest medication usage of all three groups. Patients presented with less symptoms than those in sub-phenotype 1, but ICU admittance/ death rates were higher $(31.2 \%)$.

Sub-phenotype $3(n=551)$ mostly consisted of older (median age $76[\mathrm{IQR}=69.1-81.1]$ ) males $(80.4 \%)$ with multiple comorbidities, mainly diabetes $(62.4 \%)$, hypertension (87.7\%) and other cardiovascular diseases (71.5\%), and consequent medication usage. Patients reported less symptoms such as dyspnea (67\%), headache $(8.7 \%)$ and myalgia (11.6\%). ICU admission and/or 21 -day mortality occurred in $43.2 \%$ of patients.

In parallel with Legrand et al., sub-phenotype 1 was characterized by a large percentage of women and had the most favorable outcome. Sub-phenotype 3 differentiated itself by an older age together with a higher prevalence 


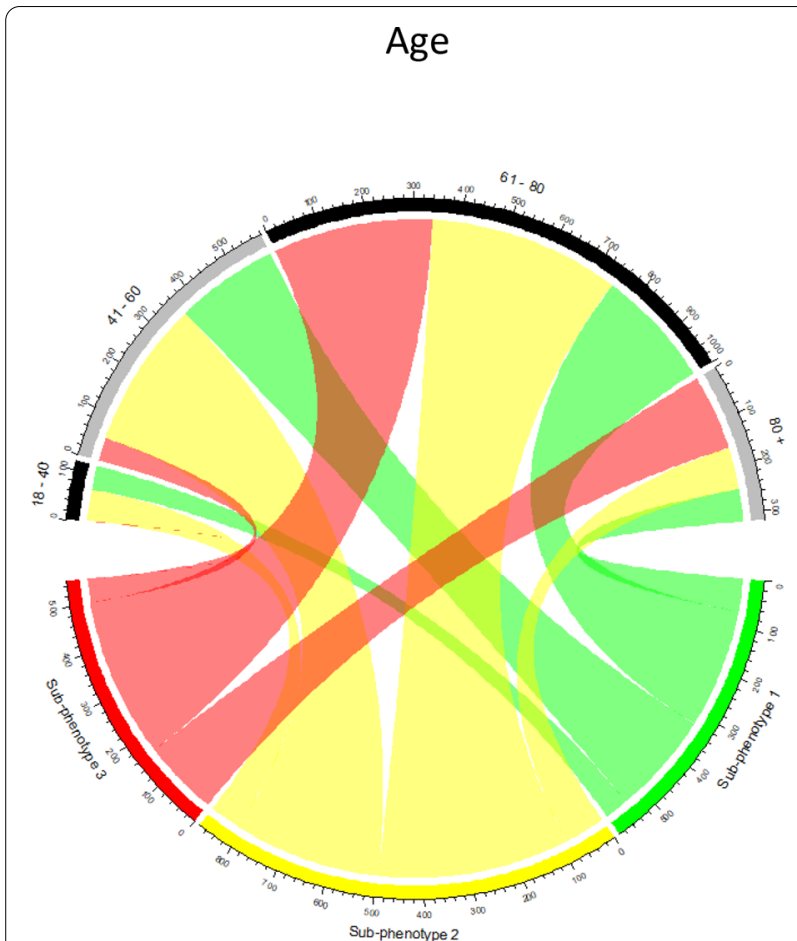

Symptoms

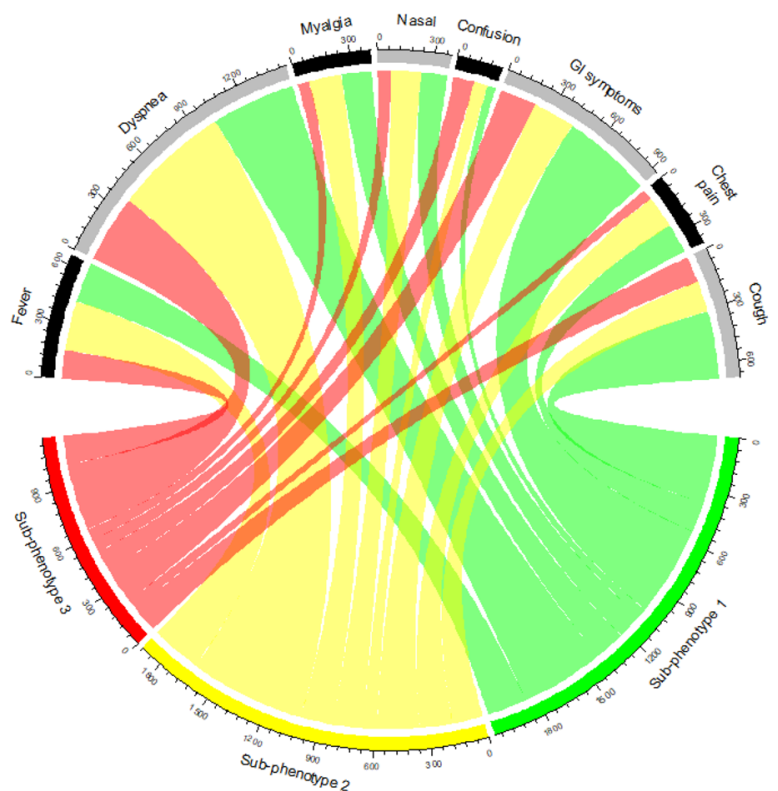

Sex

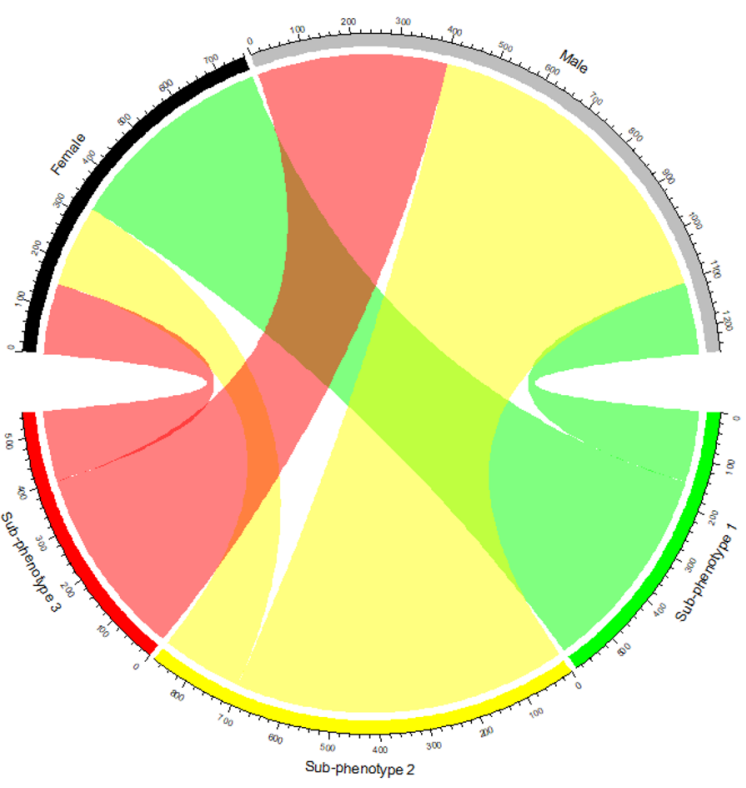

Comorbidities and medication

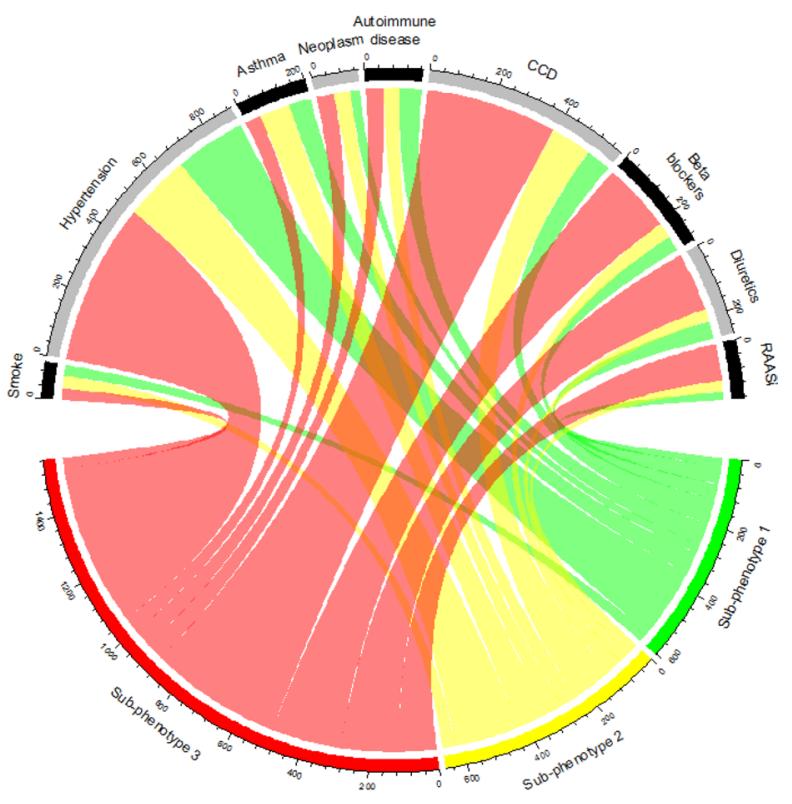

Fig. 1 Chord diagrams of the distributions of traits within the three sub-phenotypes in hospitalized patients with COVID-19. In these chord diagrams, the ribbons connect from the phenotype to the variables described. The proportion on the circle represents which group is more likely to have these characteristics traits (age, sex, symptoms, comorbidities and medication). Gl: gastro-intestinal, CCD: chronic cardiovascular disease, RAASi: renin-angiotensin-aldosterone system inhibitor 
of comorbidities and a most unfavorable outcome. The distributions of clinical characteristics were largely comparable to the original study across all sub-phenotypes. Notable differences with Legrand et al. were the relatively low age and percentages of women in sub-phenotype 2 . We speculate that some female patients who were clustered as sub-phenotype 2 in the original study were clustered into sub-phenotype 1 in our study, perhaps due to slight differences in the prevalence of baseline characteristics in our more severely ill population.

We believe the main value of these sub-phenotypes lies not with their ability to discriminate between clinical outcomes, but in their potential to understand disease heterogeneity and find more homogeneous patient subgroups that may respond more similarly to certain treatments.

In conclusion, our large multicenter cohort of hospitalized COVID-19 patients showed largely similar distributions of the characteristics as Legrand et al. found, albeit in a more severely ill population. We validated the robustness of these three clinical phenotypes, which are strongly related to clinical outcomes.

\section{Supplementary Information}

The online version contains supplementary material available at https://doi. org/10.1007/s00134-021-06363-9.

\section{Author details}

${ }^{1}$ Center of Experimental and Molecular Medicine (C.E.M.M.), Location Academic Medical Center, Amsterdam UMC, Amsterdam, The Netherlands.

${ }^{2}$ Division of Infectious Diseases, Department of Internal Medicine, Location AMC, Amsterdam UMC, Amsterdam, The Netherlands.

\section{Acknowledgements}

The members of the COVID Predict Study Group are: Renee A. Douma: Department of Internal Medicine, Flevoziekenhuis, Almere, The Netherlands. Joop $P$ van den Berg: Department of Internal Medicine, VieCurie Medical Centre, Venlo, the Netherlands. Tom Dormans: Department of Intensive Care, Zuyderland Medical Center, Heerlen, The Netherlands. Auke C. Reidinga: Department of Intensive Care, Martiniziekenhuis, Groningen, The Netherlands. Niels C. Gritters van den Oever: Department of Intensive Care, Treant Zorggroep, Emmen, the Netherlands. Peter G. Noordzij: Department of Anesthesiology and Intensive Care, St Antonius Hospital, Nieuwegein, The Netherlands. Suat Simsek: Department of Internal Medicine, Northwest Clinical, Alkmaar, The Netherlands. Bart P. A. Spaetgens: Department of Internal Medicine, Maastricht University, Maastricht University Medical Center, Maastricht, The Netherlands. Paul W.G. Elbers: EDIC, Department of Intensive Care Medicine, Research VUmc Intensive Care (REVIVE), Amsterdam Medical Data science (AMDS), Amsterdam Cardiovascular Sciences (ACS), Amsterdam Infection and Immunity Institute (AI\&II), Amsterdam UMC, location VUmc, Amsterdam, The Netherlands. Martijn Beudel: Department of Neurology, Amsterdam Neuroscience Institute, Amsterdam UMC, location AMC, Amsterdam, The Netherlands.

\section{Compliance with ethical standards}

\section{Conflicts of interest}

The authors report no conflict of interest.

\section{Publisher's Note}

Springer Nature remains neutral with regard to jurisdictional claims in published maps and institutional affiliations.

Accepted: 28 January 2021

Published online: 18 February 2021

\section{References}

1. Wiersinga WJ, Rhodes A, Cheng AC, Peacock SJ, Prescott HC (2020) Pathophysiology, transmission, diagnosis, and treatment of coronavirus disease 2019 (COVID-19): a review. JAMA 324(8):782-793

2. Data Science Collaborative Group et al (2021) Differences in clinical deterioration among three sub-phenotypes of COVID-19 patients at the time of first positive test: results from a clustering analysis. Intensive Care Med 47:113-115

3. Seymour CW et al (2019) Derivation, validation, and potential treatment implications of novel clinical phenotypes for sepsis. JAMA 321(20):2003-2017

4. Schinkel M, Virk HS, Nanayakkara PWB, van der Poll T, Wiersinga WJ (2020) What sepsis researchers can learn from COVID-19. Am J Respir Crit Care Med. https://doi.org/10.1164/rccm.202010-4023LE

5. Prokop M et al (2020) CO-RADS-A categorical CT assessment scheme for patients with suspected COVID-19: definition and evaluation original research". Radiology. https://doi.org/10.1148/radiol.2020201473 